\title{
Control Issues of an Autonomous Vehicle
}

\author{
Q. P. Ha, T. H. Tran, S. Scheding, G. Dissanayake, and H. F. Durrant-Whyte
}

\begin{abstract}
This paper addresses some control issues of a robotic amphibious vehicle that can serve as a general framework for automation of tractors used in construction. These include the vehicle's low-level dynamic equations, the development of its braking control system, kinematics in interactions with ground and the slip problem. Simulation and real-time results to date are presented.
\end{abstract}

Index Terms-Unmanned Ground Vehicle, dynamic and kinematic modelling, skid-steering, sliding mode control, slip

\section{INTRODUCTION}

$\mathrm{T}$ HE ARGO is an ongoing important project using a robotic amphibious vehicle as the experimental platform, currently developed at the ARC Centre of Excellence for Autonomous Systems (CAS). The vehicle is a fully autonomous outdoor one that can serve as a general framework for automation of tractors used in construction. The robotic vehicle is automated from a $20 \mathrm{hp}, 3 \mathrm{~m} \mathrm{x} 1.45 \mathrm{~m} \mathrm{x}$ $1.1 \mathrm{~m}, 0.5$ ton automotive amphibious vehicle that can achieve $30 \mathrm{~km} / \mathrm{h}$ on land and $3 \mathrm{~km} / \mathrm{h}$ on water. The vehicle is equipped with throttle and left/right actuators, angular speed encoders, GPS, and other sensors for control and navigation.

The vehicle, shown in Fig. 1, uses a V-twin combustion engine. Power is transmitted from the engine to the eight wheels through a continuous variable transmission (CVT), gearbox, differential and a chain system. The vehicle wheels are connected by a chain system and driven by the left and right outputs of a differential. Two brake discs are attached to the outputs of the differential and can be operated separately. The differential and braking system enables turning the vehicle (skid-steering). The driveline of the vehicle, shown in Fig. 2, consists of the engine, CVT, gearbox, differential (in gearbox), chain system, and eight wheels.

This work was supported by the ARC Centre of Excellence programme, funded by the Australian Research Council (ARC) and the New South Wales State Government.

The authors are with the ARC Centre of Excellence for Autonomous Systems (CAS). Q. P. Ha, T. H. Tran and G. Dissanayake are with, the Mechatronics and Intelligent Systems Group, a CAS partner at the Faculty of Engineering, University of Technology, Sydney (UTS), Australia (e-mail: quangha@eng.uts.edu.au, ttran@eng.uts.edu.au, gdissa@eng.uts.edu.au; Corresponding author: Q. P. Ha, phone +61-2-9514-5422; fax: +61-2-95142655).

S. Scheding and H. F. Durrant-Whyte are with the Australian Centre for Field Robotics, a CAS partner at The University of Sydney, Australia. (e-mail: steve@acfr.usyd.edu.au,hugh@acfr.usyd.edu.au).

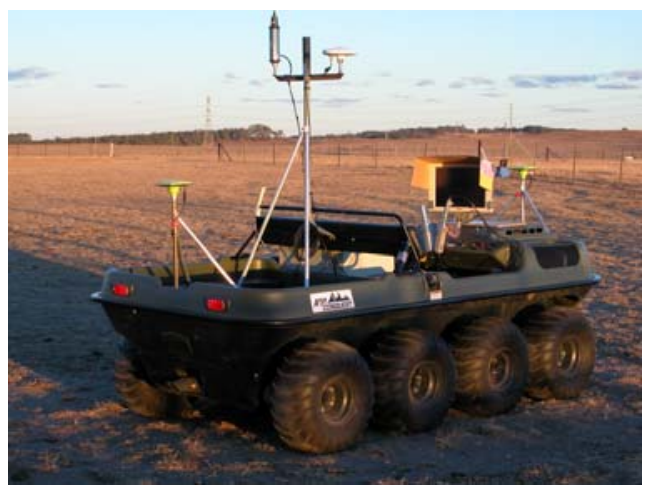

Fig.1. ARGO-a robotic amphibious vehicle

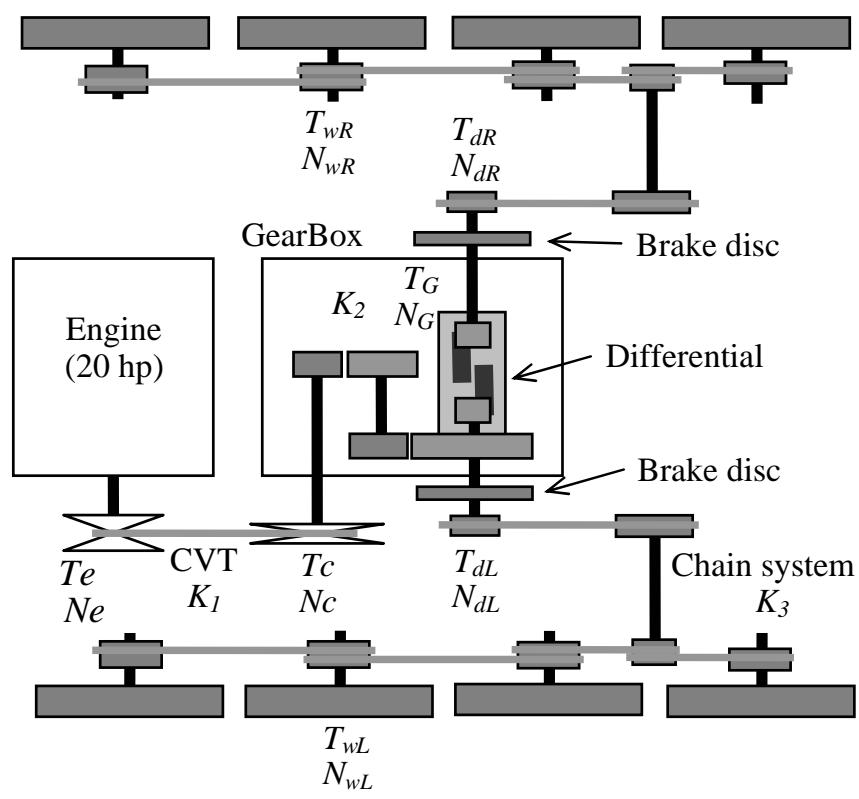

Fig.2. ARGO driveline

Several trials have been completed and experimental data were examined. The project encompasses several research areas in robotics: perception, control, planning, and system architecture. Here in this paper only machine control issues are concerned. Based on the automotive engineering theory and these trials, the vehicle's driveline, including the engine, CVT, gearbox, differential, chains and wheels, has been analysed and modelled with simulation and testing results given [1]. 
In this paper dynamics of the whole vehicle and the lowlevel control system are presented. Kinematic equations of the vehicle in interactions with ground are also derived taking into account the vehicle slip. Some preliminary results are given, with an outline on the control problems included for future work.

\section{DyNAMIC MODELLING}

Fig. 3 represents the input-output relationship of the vehicle in terms of control. The three inputs are the throttle, left and right brake voltages, $u_{t h}, u_{b L}$, and $u_{b R}$. The vehicle's two outputs are its velocity and angular velocity or left and right wheels' angular velocities. The throttle angle is controlled by a DC servo motor with the following transfer function:

$$
\theta=\frac{K_{t h}}{s\left(\tau_{t h} s+1\right)} u_{t h},
$$

where $\theta$ is throttle position, and $K_{t h}$. and $\tau_{t h}$ are the gain and time constant. The engine can be modelled by combining dynamics of its components including throttle body, intake manifold, mass flow rate, compression and torque generation [2]. For the control purpose, the generating torque, $T_{e}$, of a combustion engine can be modelled as a first-order transfer function [3]:

$$
T_{e}=\frac{K_{e}}{\tau_{p} s+1} \theta,
$$

where $K_{p}$ and $\tau_{p}$ are respectively the engine gain and time constant.

Taking into account all parts of the vehicle driveline [1], its dynamics can be written as

$$
J_{\Sigma} \dot{\omega}_{e}+b_{\Sigma} \omega_{e}=T_{e}-T_{\Sigma}-T_{\text {turn }},
$$

where $\omega_{e}$ is the engine angular speed, $J_{\Sigma}$ is the total moment of inertia, $b_{\Sigma}$ is the total damping coefficient, and $T_{\Sigma}$ is the total load torque, excluding the component arising due to turning. The turning load can be expressed as

$$
T_{\text {turn }}=K_{1} K_{2}\left(T_{b R}+T_{b L}+K x\right),
$$

where $T_{b R}, T_{b L}$ are the right, left braking torque and $K x$ is a component that is proportional to difference $x$ in speed between the case and right output of differential [1], and to turning load ratio $K$, depending on tire-terrain interactions. Note that $J_{\Sigma}, b_{\Sigma}$, and $T_{\Sigma}$ are dependent on gear ratios of CVT, $K_{1}$, of gearbox, $K_{2}$, and of the chain system, $K_{3}$, in which $K_{1}$ is a nonlinear function of the engine speed and the load on CVT [4] that can be represented as shown in Fig. 4.

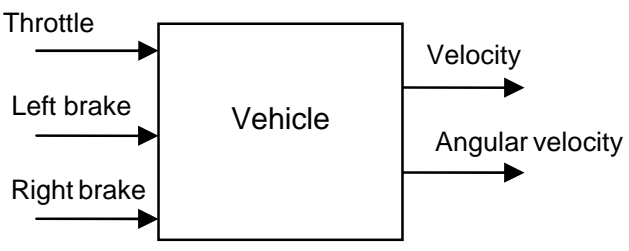

Fig. 3. ARGO input-output

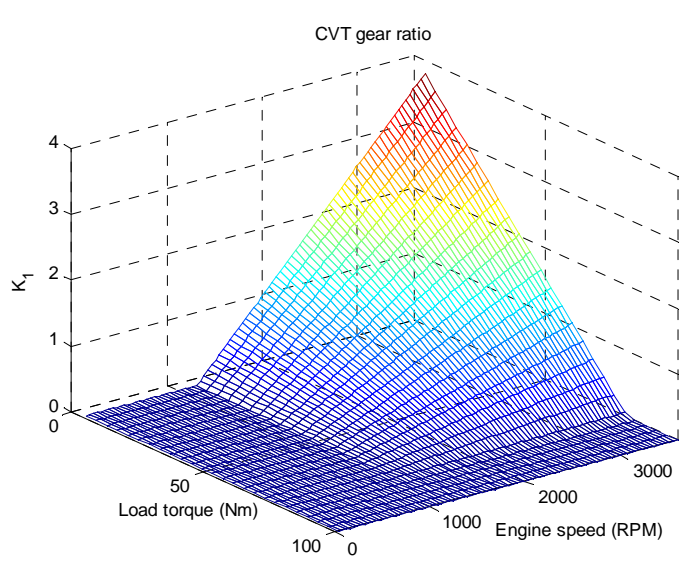

Fig. 4. CVT gear ratio

The right and left braking systems are identical and consist of a voltage to current amplifier to supply for a linear actuator, the actuator comprising a DC servo motor and a ball-screw system, and a hydraulic cylinder driven by the actuator. The (right, left) actuator can be described by the following equations

$$
\begin{aligned}
& T_{m}=K_{a} K_{i} u_{b R, L} \\
& \frac{\theta_{m}}{T_{m}}=\frac{K_{m}}{s\left(J_{m} s+B_{m}\right)},
\end{aligned}
$$

where $T_{m}, \theta_{m}$ and $u_{b R, L}$ are the motor's torque, position and the (right, left) applying voltage, $K_{a}, K_{i}$ are the voltagecurrent amplifier coefficient and motor torque coefficient, and $J_{m}, B_{m}$ are the motor's moment of inertia and viscous damping coefficient. With the relation between hydraulic pressure, $y_{R, L}$, and piston position ( $\theta_{m}$ ) being expressed as a gain $f\left(\theta_{m}\right)$ with a time constant of $\tau_{h}$, i.e.

$$
\tau_{h} \dot{y}_{R, L}+y_{R, L}=f\left(\theta_{m}\right)
$$

the braking torque can be approximated by

$$
T_{b R, L}=K_{b} y_{R, L}
$$

where the torque is considered as proportional to the pressure 
by $K_{b}$.

Choosing the state variables as $x_{1}=\theta, \quad x_{2}=\dot{\theta}$, $x_{3}=T_{e}, \quad x_{4}=\omega_{e}, \quad x_{5}=\theta_{m R}, \quad x_{6}=\omega_{m R}, \quad x_{7}=y_{R}$, $x_{8}=\theta_{m L}, \quad x_{9}=\omega_{m L}$, and $x_{10}=y_{L}$, one can obtain for the vehicle the following non-linear state equations

$$
\begin{aligned}
& \dot{x}_{1}=x_{2} \\
& \dot{x}_{2}=-\frac{1}{\tau_{t h}} x_{2}+\frac{1}{\tau_{t h}} u_{t h} \\
& \dot{x}_{3}=\frac{K_{p}}{\tau_{p}} x_{2}-\frac{1}{\tau_{p}} x_{3} \\
& \dot{x}_{4}=\frac{1}{J_{\Sigma}} x_{3}-\frac{b_{\Sigma}}{J_{\Sigma}} x_{4}-\frac{T_{\Sigma}}{J_{\Sigma}}-\frac{T_{t u r n}}{J_{\Sigma}} \\
& \dot{x}_{5}=x_{6} \\
& \dot{x}_{6}=-\frac{B_{m R}}{J_{m R}} x_{6}+\frac{K_{a} K_{i} K_{m R}}{J_{m R}} u_{b R} \\
& \dot{x}_{7}=\frac{1}{\tau_{h}} f\left(x_{5}\right)-\frac{1}{\tau_{h}} x_{7} \\
& \dot{x}_{8}=x_{9} \\
& \dot{x}_{9}=-\frac{B_{m L}}{J_{m R}} x_{9}+\frac{K_{a} K_{i} K_{m R}}{J_{m R}} u_{b L} \\
& \dot{x}_{10}=\frac{1}{\tau_{h}} f\left(x_{8}\right)-\frac{1}{\tau_{h}} x_{10} .
\end{aligned}
$$

The output right and left wheel angular velocities, $\omega_{R}$ and $\omega_{L}$, are then [1]:

$$
\begin{aligned}
& \omega_{R}=K_{3}\left(K_{2} K_{1} x_{4}-x\right) \\
& \omega_{L}=K_{3}\left(K_{2} K_{1} x_{4}+x\right),
\end{aligned}
$$

where the speed difference, $x$, is calculated as [1]:

$$
x=\frac{T_{b R}-T_{b L}}{b_{D, \text { in }}}, T_{b R}=K_{b} f\left(x_{5}\right), T_{b L}=K_{b} f\left(x_{8}\right),
$$

in which $b_{D, \text { in }}$ represents viscosity inside the differential's case.

\section{CLOSED LOOP LOW LEVEL CONTROL}

In the vehicle's state equations (8), the first two equations represent the throttle dynamics, the third one is for the engine, the fourth equation describes the vehicle motion, the next three equations are for the right brake, and the last three ones for the left brake. While controlling the throttle in closed loop is rather simple as only a small load involved, the braking control system needs however a special treatment because of high nonlinearity in the hydraulic pressure and complicated vehicle-terrain interactions. Robust control strategies would therefore be required to control the right and left braking systems.

The braking system uses two feedback loops, one from the actuator position encoder and the other from the pressure transducer. A block diagram for the system is shown in Fig. 5. Here, the cascade control principle is adopted [5]. The open loop transfer function of the inner loop-the actuator position control one- with a cascaded position controller $G_{C P}(s)$ is

$$
G_{o 1}(s)=G_{C P}(s) \frac{K_{a} K_{i} K_{m}}{s\left(J_{m} s+B_{m}\right)}
$$

As the actuator is a DC servo motor with a small inertia, choosing the smallest uncompensated time constant as $T_{\mu}=\frac{J_{m}}{B_{m}}$, the open loop transfer function (11) will take the standard symmetric optimum form [5] of $\frac{1}{2 T_{\mu} s\left(T_{\mu} s+1\right)}$, if the position controller is chosen as a proportional one with the optimum gain:

$$
G_{C P}(s)=\frac{J_{m}}{2 T_{\mu}^{2} K_{a} K_{i} K_{m}} .
$$

One can now proceed with the pressure control loop of the braking system with the inner loop replaced equivalently by the position closed loop transfer function:

$$
G_{1}(s)=\frac{\theta_{m}}{u}=\frac{1}{2 T_{\mu} s\left(T_{\mu} s+1\right)+1} \approx \frac{1}{\tau s+1} .
$$

where $u$ is the set-point input and $\tau=2 T_{\mu}$.

As the outer loop involves the highly nonlinear hydraulic pressure and external disturbance due to interactions with terrain, a sliding mode controller (SMC) is proposed here, making use of the SMC's predominant property of robustness. For this let the pressure error be defined as

$$
e(t)=y(t)-y_{r e f}(t)
$$

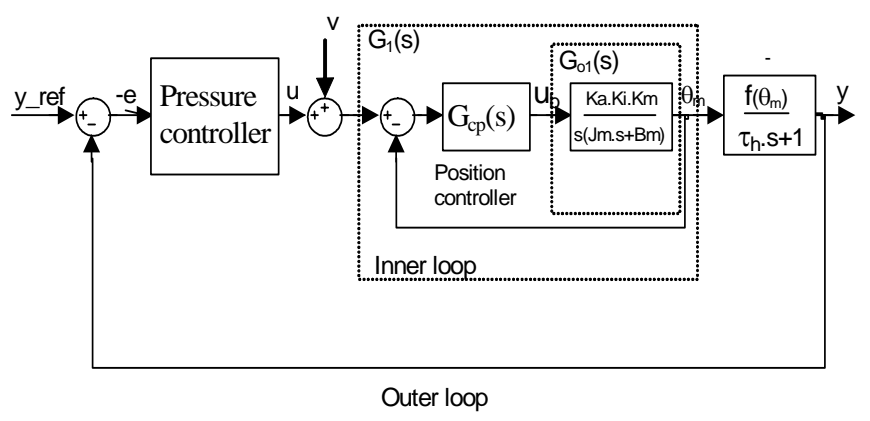

Fig. 5. Braking control system block diagram 
With a sliding function defined as $S=\dot{e}+\lambda e$, where $\lambda$ is a positive scalar to be selected, consider the Lyapunov function $V=\frac{1}{2} S^{2}$. Taking the first time derivative of $V$ yields $\dot{V}=S \dot{S}$, where

$\dot{S}=\ddot{e}+\lambda \dot{e}=-\frac{1}{\tau_{h}} \dot{y}+\frac{1}{\tau_{h}} f^{\prime}\left(\theta_{m}\right) \dot{\theta}_{m}-\ddot{y}_{r e f}+\lambda \dot{y}-\lambda \dot{y}_{r e f}$.

From (13), one can obtain $\dot{\theta}_{m}=-\frac{1}{\tau} \theta_{m}+\frac{1}{\tau}(u+v)$, where $v$ is an unknown input accounting for external disturbance, uncertainties and nonlinearities. Thus, (15) becomes

$$
\begin{aligned}
\dot{S} & =\left(\lambda-\frac{1}{\tau_{h}}\right)\left[-\frac{1}{\tau_{h}} y+\frac{1}{\tau_{h}} f\left(\theta_{m}\right)\right] \\
& +\frac{1}{\tau_{h}} f^{\prime}\left(\theta_{m}\right)\left[-\frac{1}{\tau} \theta_{m}+\frac{1}{\tau}(u+v)\right]-\ddot{y}_{\text {ref }}-\lambda \dot{y}_{\text {ref }}, \text { or } \\
\dot{S} & =\alpha e-\alpha f\left(\theta_{m}\right)-\beta\left(\theta_{m}\right) \theta_{m}+\beta\left(\theta_{m}\right)(u+v)+\varphi_{\text {ref }},
\end{aligned}
$$

where $\quad \alpha=\left(\frac{1}{\tau_{h}^{2}}-\frac{\lambda}{\tau_{h}}\right), \quad \varphi_{\text {ref }}=-\alpha y_{\text {ref }}+\lambda \dot{y}_{\text {ref }}+\ddot{y}_{\text {ref }}, \quad$ and $\beta\left(\theta_{m}\right)=\frac{1}{\tau \tau_{h}} f^{\prime}\left(\theta_{m}\right)$.

Assuming $\beta\left(\theta_{m}\right) \neq 0$, the equivalent control, $u_{e q}$, is obtained at the nominal regime $(v=0)$ from $\dot{S}=0$ :

$$
u_{e q}=\theta_{m}+\frac{1}{\beta\left(\theta_{m}\right)}\left[\alpha f\left(\theta_{m}\right)-\alpha e+\varphi_{r e f}\right] .
$$

Now for $v \neq 0$ the control law for SMC has the form of [6]:

$$
u=u_{e q}+u_{r} .
$$

Assuming $v$ is upper-bounded, $\|v\| \leq \rho$, one can easily verify that if the robust control, $u_{r}$, is chosen as

$$
u_{r}=-\rho \operatorname{sign}\left(S \beta\left(\theta_{m}\right)\right) \text {, }
$$

then the sliding condition $\dot{V}<0$ is satisfied since

$$
\begin{aligned}
\dot{V} & =S \dot{S}=S\left[\alpha e-\alpha f\left(\theta_{m}\right)-\beta \theta_{m}+\beta\left(u_{e q}+u_{r}+v\right)-\varphi_{r e f}\right] \\
& =S \beta[-\rho \operatorname{sign}(S \beta)+v] .
\end{aligned}
$$

Some SMC implementation conservatisms can be treated properly using, e.g., the techniques given in [6,7].

\section{KINEMATIC AND GROUND INTERACTION MODELLING}

In autonomous navigation of the vehicle on an unknown terrain, the vehicle slip remains an important issue. This section will be devoted to the derivation of slip velocities for this eight-wheel vehicle, and the discussion as to how to estimate them given some knowledge about the ground interaction.

Let us first consider the vehicle's kinematic diagram as shown in Fig. 6(a) with its projection on a horizontal plane shown in Fig. 6(b). Let's assume that the center of mass is displaced at distance $d$ along the centreline of the vehicle from the centroid and place the origin of coordinates at the center of mass. $Z$-axis is vertically upward and $X$-axis is along the vehicle centreline. Let $c$ be the height of the center of mass above the ground when on the level, $a$ - the longitudinal distance between successive axles, and $2 b$ - the vehicle track width.

Positions of contact points of wheels $1,2, \ldots$, and 8 in relation to the vehicle frame are respectively

$\mathbf{P}_{\mathbf{1}}=(3 / 2 a-d) \mathbf{i}+b \mathbf{j}-c \mathbf{k}, \quad \mathbf{P}_{2}=(3 / 2 a-d) \mathbf{i}-b \mathbf{j}-c \mathbf{k}$,

$\mathbf{P}_{3}=(1 / 2 a-d) \mathbf{i}+b \mathbf{j}-c \mathbf{k}, \mathbf{P}_{4}=(1 / 2 a-d) \mathbf{i}-b \mathbf{j}-c \mathbf{k}$,

$\mathbf{P}_{5}=-(1 / 2 a+d) \mathbf{i}+b \mathbf{j}-c \mathbf{k}, \quad \mathbf{P}_{6}=-(1 / 2 a+d) \mathbf{i}-b \mathbf{j}-c \mathbf{k}$,

$\mathbf{P}_{7}=-(3 / 2 a+d) \mathbf{i}+b \mathbf{j}-c \mathbf{k}, \mathbf{P}_{\mathbf{8}}=-(3 / 2 a+d) \mathbf{i}-b \mathbf{j}-c \mathbf{k}$.

Let the velocity of the centre of mass relative to a fixed frame coincident with the vehicle frame be $\mathbf{V}=\mathrm{V}_{\mathrm{x}} \mathbf{i}+\mathrm{V}_{\mathrm{y}} \mathbf{j}$, the angular velocity be $\Omega=\Omega \mathbf{k}$, the angular velocity of the leftside wheels be $\omega_{\mathrm{L}}=\omega_{\mathrm{L}} \mathbf{j}$, and that of the right-side wheels be $\omega_{\mathbf{R}}=\omega_{\mathrm{R}} \mathbf{j}$.

Slip velocity, $\mathbf{V}_{\mathbf{s} 1}$ of wheel 1 is the velocity of contact point of wheel 1 to ground:

$$
\begin{aligned}
\mathbf{V}_{\mathrm{s} \mathbf{1}} & =\mathbf{V}+\Omega \mathbf{k} \times \mathbf{P}_{\mathbf{1}}+\omega_{\mathrm{L}} \mathbf{j} \times(-r \mathbf{k}) \\
& =\mathrm{V}_{\mathrm{x}} \mathbf{i}+\mathrm{V}_{\mathrm{y}} \mathbf{j}+\Omega \mathbf{k} \times((3 / 2 a-d) \mathbf{i}+b \mathbf{j}-c \mathbf{k})-r \omega_{\mathrm{L}} \mathbf{i} \\
& =\left(\mathrm{V}_{\mathrm{x}}-b \Omega-r \omega_{\mathrm{L}}\right) \mathbf{i}+\left(\mathrm{V}_{\mathrm{y}}+(3 / 2 a-d) \Omega\right) \mathbf{j} .
\end{aligned}
$$

Hence, the longitudinal and lateral slip velocities of wheel 1 are respectively

$$
\mathrm{V}_{\mathrm{sx} 1}=\mathrm{V}_{\mathrm{x}}-b \Omega-r \omega, \quad \mathrm{V}_{\mathrm{sy} 1}=\mathrm{V}_{\mathrm{y}}+(3 / 2 a-d) \Omega .
$$

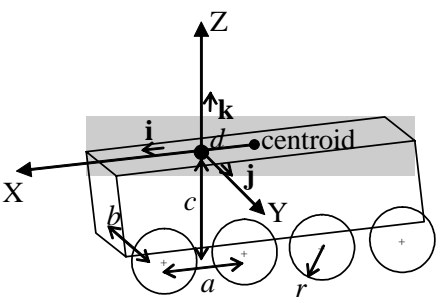

(a)

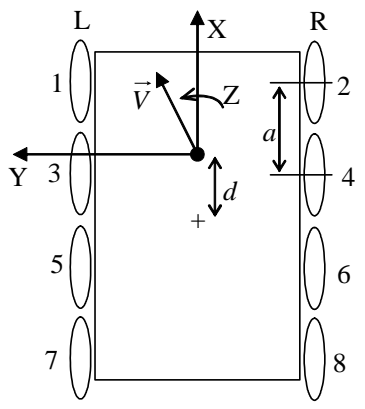

(b)
Fig. 6. ARGO kinematic diagrams 
Similarly, one can derive

$$
\begin{aligned}
\mathrm{V}_{\text {sxi }} & =\mathrm{V}_{\mathrm{x}}+(-1)^{i} b \Omega-r \omega, i=1,2, \ldots, 8 . \\
\mathrm{V}_{\text {sy } 1} & =\mathrm{V}_{\text {sy } 2}=\mathrm{V}_{\mathrm{y}}+(3 / 2 a-d) \Omega \\
\mathrm{V}_{\text {sy } 3} & =\mathrm{V}_{\text {sy } 4}=\mathrm{V}_{\mathrm{y}}+(1 / 2 a-d) \Omega \\
\mathrm{V}_{\text {sy } 5} & =\mathrm{V}_{\text {sy } 6}=\mathrm{V}_{\mathrm{y}}-(1 / 2 a-d) \Omega \\
\mathrm{V}_{\text {sy } 7} & =\mathrm{V}_{\text {sy } 8}=\mathrm{V}_{\mathrm{y}}-(3 / 2 a-d) \Omega .
\end{aligned}
$$

Now given the angular velocities $\omega_{L}, \omega_{R}$ of the left-, rightside wheels, if the reaction force on wheel $i$ is known as $\mathbf{F}_{\boldsymbol{i}}=$ $F_{x i} \mathbf{i}+F_{y i} \mathbf{j}+F_{z i} \mathbf{k},\left(F_{z i}>0\right)$, then one shall be able to estimate slip velocities (23). Practically, a slip model is generally used for this purpose. For wheel $i$ let's assume the model [8]:

$$
\frac{F_{x i}}{F_{z i}}=\mu_{t i}=A_{t}+B_{t} S_{t i} \text {, and } \frac{F_{y i}}{F_{z i}}=\mu_{l i}=A_{l}+B_{l} S_{l i},
$$

where the tangential and lateral slip ratios of wheel $i$ are defined respectively as

$$
S_{t i}=\frac{V_{s x i}}{r \omega_{i}} \text { and } S_{l i}=\frac{V_{s y i}}{r \omega_{i}},
$$

and coefficients $A_{t}, B_{t}, A_{l}$, and $B_{l}$ are terrain parameters depending on the soil nature. From the Newton's law

$$
\sum_{i=1}^{8} F_{X i}=m a_{X}, \quad \sum_{i=1}^{8} F_{Y i}=m a_{Y}, \quad \sum_{i=1}^{8} F_{Z i}=m g,
$$

a set of equations can be formulated to solve explicitly for the accelerations $a_{X}, a_{Y}$, and $\dot{\Omega}$. This gives a basis for computing recursively the values of velocities $V_{X(n+1)}, V_{Y(n+1)}, \Omega_{(n+1)}$ at time step $n+1$ from those $V_{X n}, V_{Y n}, \Omega_{n}$ at step $n$ if $\omega_{\mathrm{L}}, \omega_{\mathrm{R}}$, and $A_{t}, B_{t}, A_{l}, B_{l}$ are known.

Note that on hard surfaces $A_{t}$ and $A_{l}$ may be neglected. Slip is primary due to tire tread compression and so the constants $B_{t}$ and $B_{l}$ can be measured experimentally and used consistently. On soft soil slip is however primary due to soil deformation and $A_{t}$ and $A_{l}$ are significant. Estimating appropriate values of $A_{t}, B_{t}, A_{l}$, and $B_{l}$ for this case may be problematic. On the other hand if the longitudinal and lateral components of the total reaction force (25) can somehow be estimated then the same procedure can help identify terrain parameters.

\section{SOME RESULTS AND FUTURE WORK}

A series of tests on the vehicle have been conducted for its characterization and proof of autonomy. The project is at this stage still under some refinements and further development, particularly in the vehicle control. Some results to date are given in this section. To illustrate skid steering, Fig. 7 shows typically experimental results when the vehicle was turning left. At an open-loop throttle voltage $4.5 \mathrm{~V}$, the responses of pressure, position and force of right and left brakes are shown in Fig. 7(a) and of engine and gearbox speeds in Fig. 7(b).
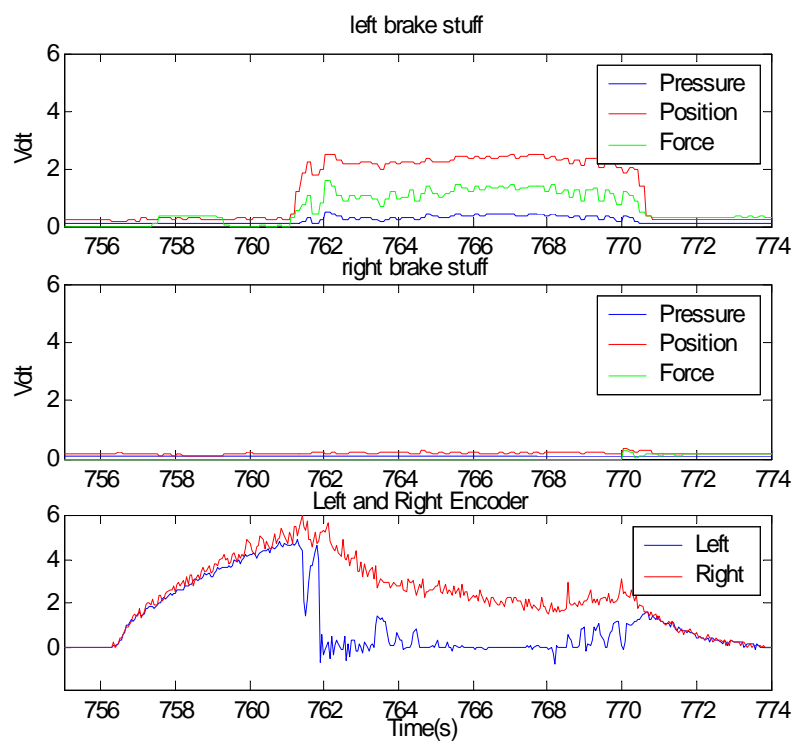

(a)
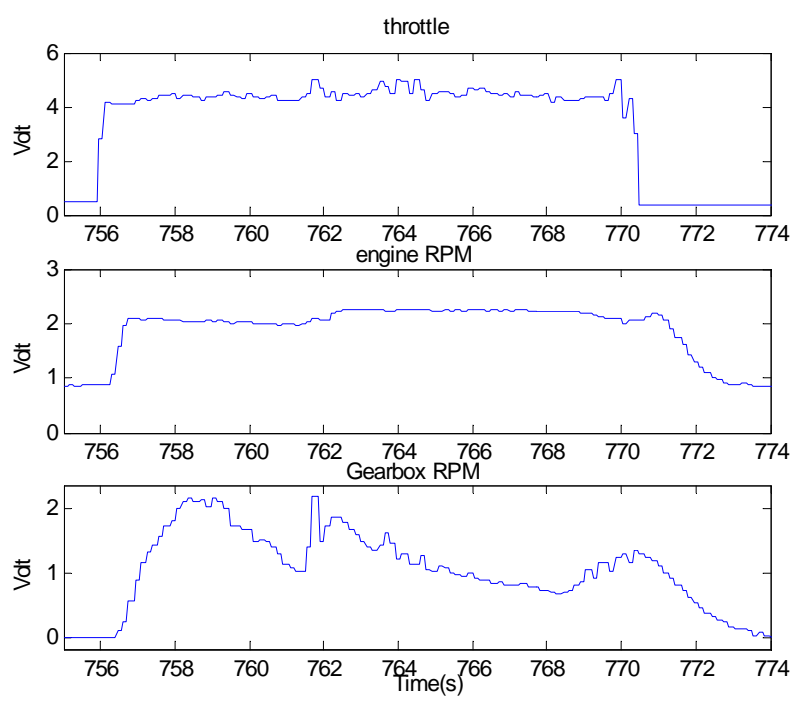

(b)

Fig. 7. Left turn at throttle $4.5 \mathrm{~V}$

Characterising tests have been compared to refine the modeling of the vehicle. Based on the driveline models and the state equations (8) a simulator has been developed using step inputs of throttle and pulses of left and right brakes for straight running and turning of the vehicle. Simulation results are shown in Fig. 8(a) for throttle, engine torque and speed, and gearbox input speed; Fig. 8(b) for left and right brakes and wheel speeds; and Fig. 8(c) for loads at wheel shafts, gearbox, and engine. These input patterns of throttle, left and right brakes are required for the vehicle to perform a desired trajectory as shown in Fig. 9. The vehicle begins at the point $(0,0)$ and runs straight in nearly 10 seconds before the first turning in a prescribed pattern of the throttle and the two brakes to trace figuratively the letters C.A.S. 

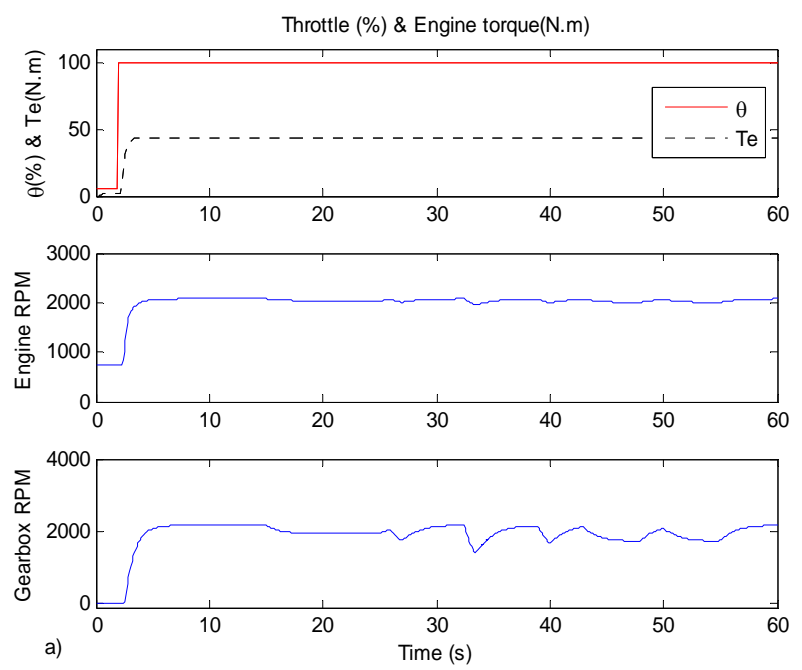

(a)
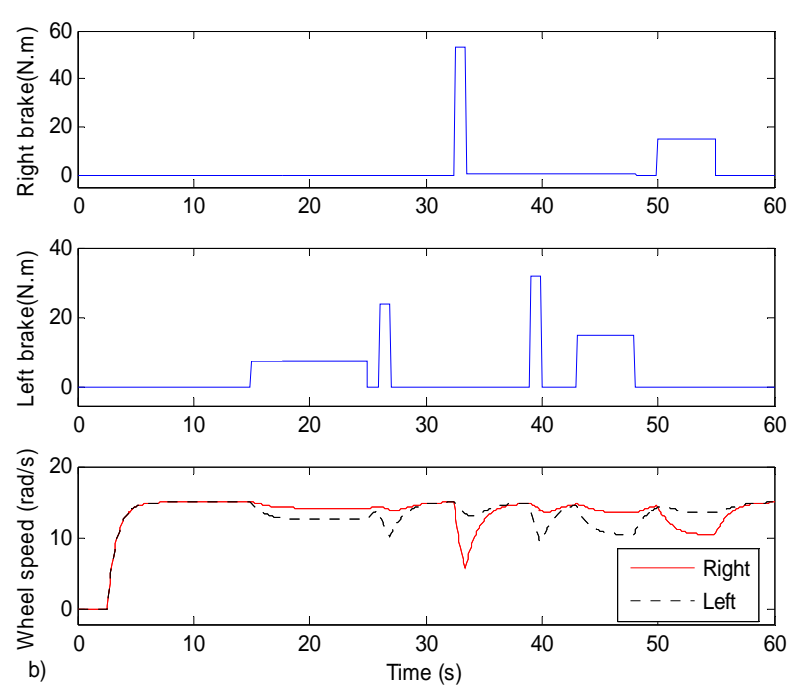

(b)
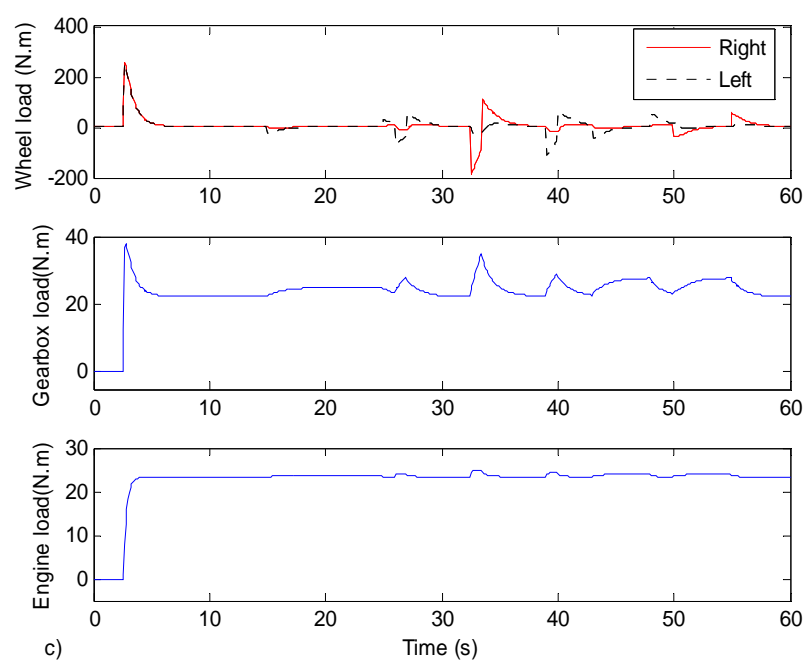

(c)

Fig. 8. Input patterns and vehicle responses

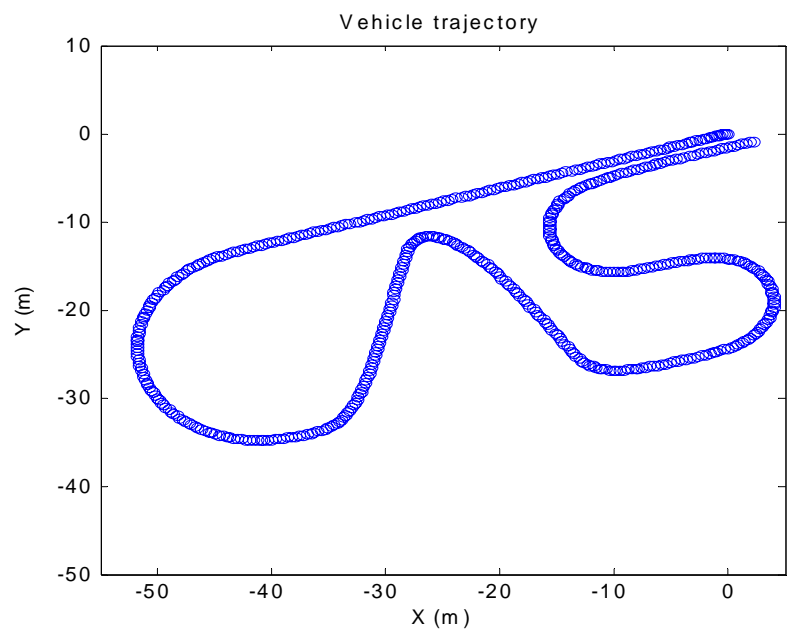

Fig. 9. Trajectory of the vehicle

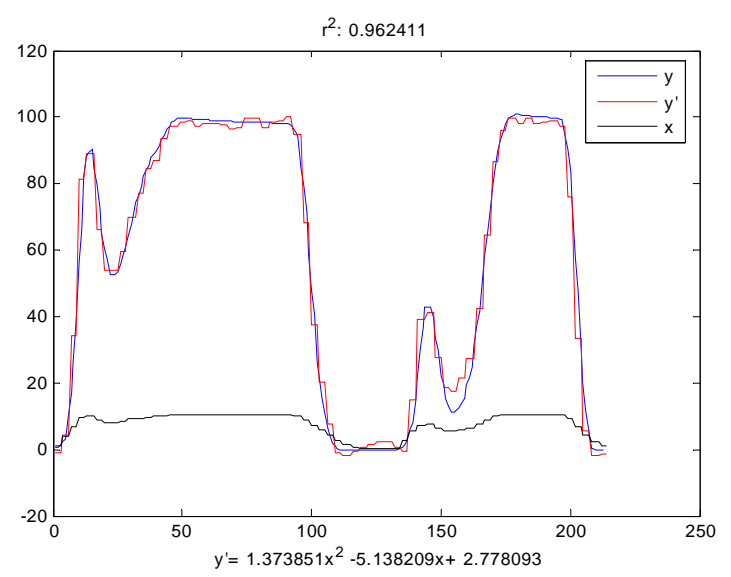

Fig. 10. Pressure function approximation

While braking is the key issue for vehicle steering, the relationship between the hydraulic pressure $y$ on the braking discs and the piston position $x=\theta_{m}$ remains highly nonlinear, making it difficult to model and control the vehicle's turning and steering. Here, after collecting experimentally data sets the least square method [9] was used to approximate the function $f(x)$ given in (6), as shown in Fig. 10. Performance of the SMC for the braking system is then illustrated in Fig. 11 where the response to, typically, $100 \%$ of pressure exhibits a fast response with no overshoot. Simulation has verified a consistent insensitivity of the control performance even in the presence of an external disturbance of up to $\pm 1000 \mathrm{~N}$. Figure 12 shows the designed controller out-performances, in terms of robustness, a standard PID pressure control loop in terms of tracking when the pressure set-point is required to follow a sinusoidal function.

Work is in progress for implementation of robust controllers for low-level control and path planning of the vehicle. Future focuses will be on interactions of the vehicle and terrain, its parameter identification, and high-level control architecture. 


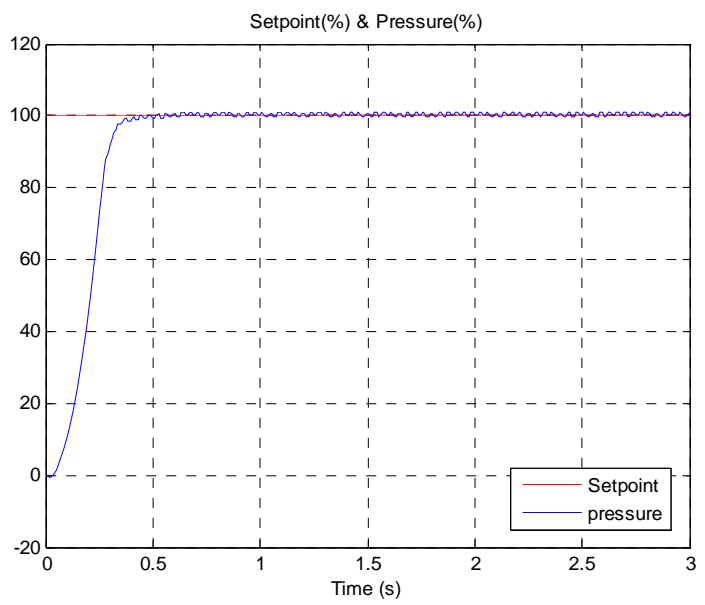

Fig. 11. SMC braking pressure response: load-insensitivity
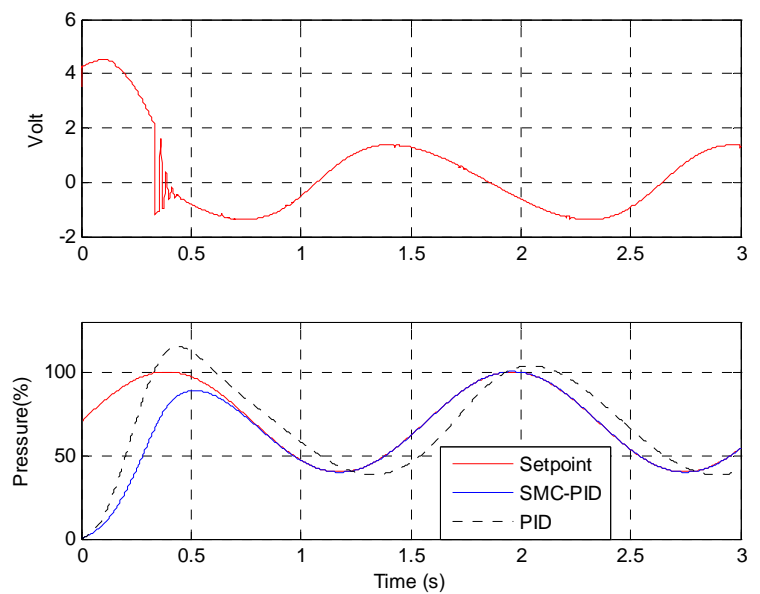

Fig. 12. SMC braking pressure response: tracking performance

\section{CONCLUSION}

We have introduced the on-going development of an autonomous vehicle, currently attracting a great deal of research effort at CAS. The unmanned ground vehicle is believed to have, together with other application areas, a certain potential in construction automation. We have presented some control issues when automating the vehicle. Its dynamics modelling has been described with the derivation of a set of state equations. The closed loop low-level control problem has been addressed, stressing on the development of a sliding mode controller for the braking system. Equations for the vehicle's slip velocities have been derived, with suggestions made on the estimation of slip velocities and terrain parameters. Some experimental and simulation results to-date were included together with an outline of future work.

\section{ACKNOWLEDGMENT}

The authors would like to thank Professor Kenneth J. Waldron for discussion and the derivation of the vehicle's slip velocities.

\section{REFERENCES}

[1] T. H Tran, Q. P. Ha, R. Grover, and S. Scheding, "Modelling of an autonomous amphibious vehicle," Proceedings of the Australiasian Conference on Robotics and Automation, pp. 1-7, Canberra 2004.

[2] P. R. Crossley and J. A. Cook, "A nonlinear model for drivetrain system development," Proc. IEE International Conference 'Control 91', Conference Publication 332, vol. 2, pp. 921-925. Edinburgh, U.K, 1991.

[3] R. Zanasi, A. Viscontit, G. Sandoni, and R. Morselli, "Dynamic modeling and control of a car transmission system", IEEE/ASME International Conference on Advanced Intelligent Mechatronics. Proceedings, pp. 416 - 421, vol.1, July 2001.

[4] P. Setlur, J. R. Wagner, D. M. Dawson, and B. Samuels, Nonlinear "Control of a Continuously Variable Transmission (CVT)", IEEE Transactions on Control Systems Technology, pp 101-108, vol. 11, no. 1, January 2003.

[5] I. D. Landau, and A. Voda, "A Method for the Autocalibration of PID Controllers", Automatica, Vol. 31. No. 1, pp. 41-53, 1995

[6] Q. P. Ha, Q. H. Nguyen, D. C. Rye, and H. F. DurrantWhyte, "Impedance control of a hydraulically-actuated robotic excavator," Automation in Construction, Vol. 9, pp. 421-435, 2000.

[7] Q. P. Ha, T. H. Tran, and H. Trinh, "Observer-based output feedback variable structure control with Application to a Two-Link Manipulator," Preprints of the 3rd IFAC Symposium on Mechatronic Systems, Sydney, Australia, September 2004, pp. 133-138.

[8] J. Y. Wong, Theory of ground vehicles, John Wiley \& Sons, 2003.

[9] Y. Maeda and Y. Kanata, "An algorithm for a leastsquare approximation problem of unknown systems," International Conference on Industrial Electronics, Control and Instrumentation, pp. 1881-1886, 1991. 